\title{
Observed Area Under the Curve Infinity
}

National Cancer Institute

\section{Source}

National Cancer Institute. Observed Area Under the Curve Infinity. NCI Thesaurus. Code C85761.

The area under the curve (AUC) extrapolated to infinity from dosing time, based on the last observed concentration. 